\title{
Traumatic events take their toll on mental health
}

\section{Results of violence and natural disasters could overwhelm services in developing world.}

Sir - Your recent News Feature "Asia's tigers get the blues" (Nature 429, 696-698; 2004) notes that levels of depression and suicide are rapidly increasing in East Asia. The breakdown of traditional community structures, the loss of family-support systems, long-distance immigration and economic uncertainty all take their toll, in the form of a rise in mental disorders. As noted in the News Feature, this cocktail of psychological stresses leads to depression, substance abuse, eating disorders and other stress-related problems.

There is, however, another important reason for the rise of depression and other mental disorders in China. During the past year, almost half a billion people there have been affected by natural disasters such as floods and earthquakes, which have caused more than 2,000 deaths and the displacement of some seven million people, according to the World Health Organization (see www.who.int/disasters/ country.cfm? countryID=10\&DocType $=2$ ).

As the number of disasters - both natural and manmade - increases around the world, we can expect to see more stress-related disorders in the twenty-first century. Post-traumatic stress disorder (PTSD), in particular, can be caused by natural disasters or by the wars and acts of violence that afflict many parts of the world. Traumatic events do not discriminate on the grounds of culture, race, gender or age (see, for example, I. Derluyn et al. Lancet 363, 861-863; 2004). And people exposed to such events are far more likely to develop psychiatric disorders such as substance abuse, major depression, PTSD and psychosomatic illness (P. P. Schnurr et al. Science 303, 168-169; 2004).

The impact of PTSD is especially devastating in developing countries, where health services are helpless to cope with the flood of traumatized people seeking refuge and help.

\section{Menachem Ben-Ezra}

Department of Psychology, Sharet Building, Tel Aviv University, Ramat-Aviv,

PO Box 39040, Tel Aviv 69978, Israel

\section{OvaCheck: let's not dismiss the concept}

Sir - Your News Feature "Running before we can walk?" (Nature 429, 496-497; 2004) highlights concerns about a new proteomic test for ovarian cancer known as OvaCheck. This test measures breakdown products of proteins in blood serum and looks for patterns that may indicate disease. The appeal of the method, now under scrutiny, derives from its simplicity and potential power; the total number of breakdown products could eventually exceed the number of gene transcripts and proteins, and may therefore provide a subtler correlation between biological events and disorders than regular expression genomics and proteomics. This approach should not be dismissed before it has been fully investigated with the best possible technological means and expertise, and under rigorously standardized conditions.

Experts quoted in the story cite concerns about mass spectrometry, biostatistics, and also environmental, dietary and psychological factors that could produce artefacts in the data. In our experience, serum preparation, handling and storage could be the largest source of artefact in mass-spectrometry readings, especially for archived samples. We are currently investigating the effects of, for instance, different blood-collection tubes and the number of freeze/thaw cycles.

The whole concept is now in danger of being declared null and void, both by some who previously embraced it and by others who lack the adequate experimental experience to participate in the debate. Although unbiased, your News Feature is likely to embolden the critics and reinforce the bleak view of this particular type of proteomic analysis, and perhaps of proteomic profiling in general.

No final assessment can be made at this point of the concept underlying the OvaCheck test. We hope that leaders from the clinical and analytical-chemistry communities will come together and make a concerted effort to refine the procedures before testing them in carefully designed clinical studies. Only then can we establish whether this approach can disclose hidden patterns of disease or whether it is the molecular equivalent of the Emperor's new clothes.

Josep Villanueva, Paul Tempst Protein Center and Molecular Biology Program, Memorial Sloan-Kettering Cancer Center, 1275 York Avenue, New York, NY 10021, USA

\section{OvaCheck: doubts voiced soon after publication}

Sir - Your News Feature "Running before we can walk?" (Nature 429, 496-497; 2004) summarizes recent developments in the use of serum proteomic patterns, in particular the OvaCheck test, for early cancer detection. It states that "the first criticisms of OvaCheck hit the public domain in June 2003".

Unfortunately, this is not accurate. Following publication of the first paper by Petricoin and colleagues on ovarian cancer (your ref. 1), The Lancet published several correspondences critiquing the method in July 2002 (Lancet 360, 169-170; 2002). More importantly, the limitations of this approach are not restricted to those revealed by the new bioinformatic analyses described in the News Feature. (For more information, see E. P. Diamandis Mol. Cell Proteomics 3, 367-378; 2004.) The cautionary notes of many authors on the limitations of this technology are useful in tempering the original optimism that this method will revolutionize the way we diagnose cancer in the future.

\section{Eleftherios P. Diamandis}

Department of Pathology and Laboratory Medicine, Mount Sinai Hospital, 600 University Avenue,

Toronto, Ontario M5G1X5, Canada

\section{Editing for posterity gets red card in Real world}

Sir — I read with amusement your News story "'Inspirational' leader quits Madrid heart project" (Nature 430, 127; 2004). Reporting a comment that the Spanish government should pay more to attract high-profile scientists, as Real Madrid did to attract David Beckham, you explained "Beckham is a footballer who left Manchester United for Real Madrid in 2003".

After discounting the possibility that newly arrived aliens were the target audience, I conclude that either the article's editor needs to get out more, or there is an institutional belief that serious subscribers to Nature cannot possibly find time for a life between the weekly arrivals of your journal, or both.

Derek Partridge

Department of Computer Science, Harrison Building, University of Exeter, Exeter EX4 4QF, UK

\section{correspondence}

Contributions to Correspondence may be submitted to corres@nature.com. They should be no longer than 500 words, and ideally shorter. 